\title{
Integrating Ultra-Wideband and Free Space Optical Communication for Realizing a Secure and High Throughput Body Area Network Architecture Based on Optical Code Division Multiple Access
}

Jawad Mirza

HITEC University

Waqas Imtiaz

UET: University of Engineering and Technology

Salman Abdul Ghafoor ( $\nabla$ salman.ghafoor@seecs.edu.pk)

National University of Sciences and Technology https://orcid.org/0000-0002-1031-4471

\section{Research Article}

Keywords: Optical body area network, Ultra-wideband, Free space optics, Optical code division multiple access

Posted Date: February 12th, 2021

DOl: https://doi.org/10.21203/rs.3.rs-189157/v1

License: (c) (1) This work is licensed under a Creative Commons Attribution 4.0 International License.

Read Full License 


\title{
Integrating Ultra-Wideband and Free Space Optical Communication for Realizing a Secure and High Throughput Body Area Network Architecture Based on Optical Code Division Multiple Access
}

\author{
Jawad Mirza, Waqas Ahmad and Salman \\ Ghafoor
}

Received: date / Accepted: date

\begin{abstract}
Nodes in a body area network (BAN) are miniature wearable or implantable battery-powered wireless sensors which continuously transmit real time vital physiological data of a patient to remote health-care center while remaining in close proximity to the human body. Therefore, BAN nodes should have the features of high data rates and low transmit powers in order to protect the human body, environment and bio-medical equipment from harmful exposure of electromagnetic radiations and electromagnetic interference (EMI). Ultra-wideband (UWB) signals have low allowable transmission power and high data rates. Therefore, we propose a low cost, low powered and secure optical body area network (OBAN) composed of four UWB-BAN nodes each transmitting at a data rate of $30 \mathrm{Mbps}$. At the control node, UWB signals from UWB-BAN nodes are encoded using spectral amplitude coding-optical code division multiple access (SAC-OCDMA) scheme and the combined signal is transmitted over free space optics (FSO) channel towards remote health-care center. At the health-care center, the combined signal is decoded and UWB signal of each UWB-BAN node is photodetected for analysis of patient's data. Log-Normal channel model is considered between control node and the health-care center. The signal received from each UWB-BAN after propagation through the FSO channel is analyzed through Bit error rate (BER) results. It was observed that the proposed architecture requires the UWB-BAN nodes to have low receiver sensitivities with the added benefits of cost-efficiency and data security.
\end{abstract}

Jawad Mirza

Electrical Engineering Department,HITEC University, Taxila, Pakistan

E-mail: 15phdee001@hitecuni.edu.pk

Waqas Ahmad

Electrical Engineering Department, University of Engineering and Technology, Peshawar, Pakistan

E-mail: waqasahmed15@gmail.com

Salman Ghafoor (Corresponding author)

School of Electrical Engineering and Computer Science, National University of Sciences and Technology (NUST), Sector H-12, Islamabad, Pakistan

E-mail: salman.ghafoor@seecs.edu.pk 
Keywords Optical body area network · Ultra-wideband · Free space optics · Optical code division multiple access

\section{Introduction}

Body area network (BAN) is a cutting-edge and promising wireless technology operating at the close proximity to human body which enables wireless communications between on-body sensors called BAN nodes and a control node usually mounted on human body or located at a fixed distant place near to human body $[1,2]$. This control node acts as a coordinator establishing communication link with all BAN nodes as well as with other wireless or wired networks. One main application of BAN is the tele-medicine and e-health platforms because BAN can provide continuous remote monitoring of patients having multiple diseases and thus early detection of abnormal health conditions [3]. By timely detection of abnormal health conditions, BAN can prevent diseases and thus allow reducing health-care expenditures which is a significant challenge nowadays due to increasing percentage of aging population. Besides, the workload of medical staff can be decreased by integrating BAN with medical centers thus resulting in higher efficiency of medical staff and reduction in health-care expenditures as well.

While designing a BAN, it is of prime importance that the network designer should choose such a wireless technology which promises high capacity of information transmission between on body BAN nodes and control node along with low power spectral density. Recent studies [4] show that the electromagnetic interference (EMI) between RF devices and medical equipment, such as in hospitals could be critical. Moreover, prolong RF exposure to human body is undesirable. Therefore, it is imperative to use minimum transmit power BAN nodes on patient body so that the adverse effects of RF exposure to the body and EMI between RF devices and medical equipment can be mitigated. UWB is an attractive and promising wireless communication technique that provides carrier-less, high-data rate transmission [5]. Due to very low-radiated power which is equal to $-41.3 d B_{m} / M H z$, UWB can be a potential applicant in BAN technology which can resolve the issues of adverse effects of RF exposure to human body and EMI between RF devices and medical equipment.

Future BANs should have the features of high data rate, immunity to EMI, environmental friendly, license-free spectrum, low installation and maintenance costs. Realization of these characteristics of future BANs by employing free space optics (FSO) technology transforms them into optical body area networks (OBANs). FSO communications can transmit high capacity wireless signals while maintaining cost-efficiency due to reduced deployment costs [6,7]. Furthermore, a large license-free spectrum is offered by FSO that is immune to EMI, is environmental friendly and has high security [7]. Owing to these interesting features of FSO, FSO based OBANs are the future of BANs. Despite having multiple attractions, FSO technology is potentially prone to eavesdropping as an attacker can easily get and interpret the modulated light signals propagating through free space with little effort. OBAN is a recent research direction, therefore considerable research work has not been done over it so far. There are some pioneer research contributions $[2$, $3,8,9]$ which mainly cover mathematical aspects of OBANs including FSO channel modeling employed in OBANs, error probability, blocking effects and coding 
schemes. It is pertinent to mention that the field of OBAN is almost un-explored. This paper proposes an OBAN architecture that is based on multiple UWB-BAN nodes, each transmitting the patient physiological data at $30 \mathrm{Mbps}$ over an additive white Gaussian noise (AWGN) wireless channel to a fixed control node located in the patient's room. At the control node, a continuous wave (CW) laser array is employed to generate multiple wavelengths which are used to encode the UWB signals received at control node coming from UWB-BAN nodes using spectral amplitude coding-optical code division multiple access (SAC-OCDMA) scheme. The combined signal encoded by SAC-OCDMA scheme is then transmitted over FSO link of $0.5 \mathrm{~km}$ length towards remote healthcare center where the combined signal is decoded and each UWB signal is photo-detected followed by demodulation and performance analysis.

SAC-OCDMA technique is adapted to provide robust transmission and mitigation of the various adverse effects between the transmitter and receiver modules. Working principle of SAC-OCDMA system encompasses the translation of binary 1 s or 0 s into spectral domain by allowing or blocking desired bits through special arrangement of filters in the encoder module [10,11]. This generates multiple carriers to communicate the user information with relatively high spectral efficiency and built-in security [10]. The family of spectral amplitude codes primarily includes two types of codes namely (i) zero-cross correlation (ZCC) and (ii) fixed in-phase cross correlation codes $[11,12]$. Both are developed to mitigate the effects of multiple access interference (MAI) along with the associated phase induced intensity noise (PIIN) for non-coherent sources and optical beat interference for coherent sources at the receiving photo-detector through an efficient combination of code length, weight and cross-correlation $[11,10]$. The prior offers efficient performance for low cardinality systems by completely removing the effects of MAI with relatively simple architecture at the encoder and decoder modules. ZCC codes, as the name suggests, offers zero cross correlation between adjacent codes, which significantly elevates performance of the system through recovery of the intended spectrum with maximum auto-correlation and zero cross-correlation between the intended and interfering subscribers, respectively.

Consequently, this work adapts ZCC code called double weight zero crosscorrelation (DW-ZCC) to effectively communicate the intended signals between the control and remote health-care center and mitigate the environmental effects with added security. The proposed DW-ZCC code is constructed with reduced weight $(\mathrm{W}=2)$ in order to decrease the number of filters and elevate feasibility of the proposed setup in terms of implementation complexity and cost. Additionally, highly acknowledged direct detection technique is used to recover the intended spectrum with desired correlation properties, thereby eliminating the need for an extra arrangement in lower arm of the decoding module [11]. Another advantage of the DW-ZCC code in comparison with the conventional scheme is simplicity in code construction and support for large cardinality in relatively short reach systems $[11,13]$.

The novel contributions of our work in the field of OBANs are summarized below:

- A cost efficient encoding scheme based on SAC-OCDMA is implemented for OBANs by employing multiple wavelengths generated by a single $\mathrm{CW}$ laser array. 
- Deployment and maintenance costs are reduced by employing FSO link instead of fiber or wireless link between control node and remote health-care center.

- EMI between UWB-BAN nodes and medical equipment as well as RF exposure of UWB-BAN to human body is reduced by employing low transmit power UWB wireless technology.

The proposed architecture is best suited for implementation of e-health and tele-medicine platforms in all kind of assisted living facilities and hospitals. The simulations are implemented by using Optisystem 16 commercial tool.

\section{Application Scenario of Proposed OBAN}

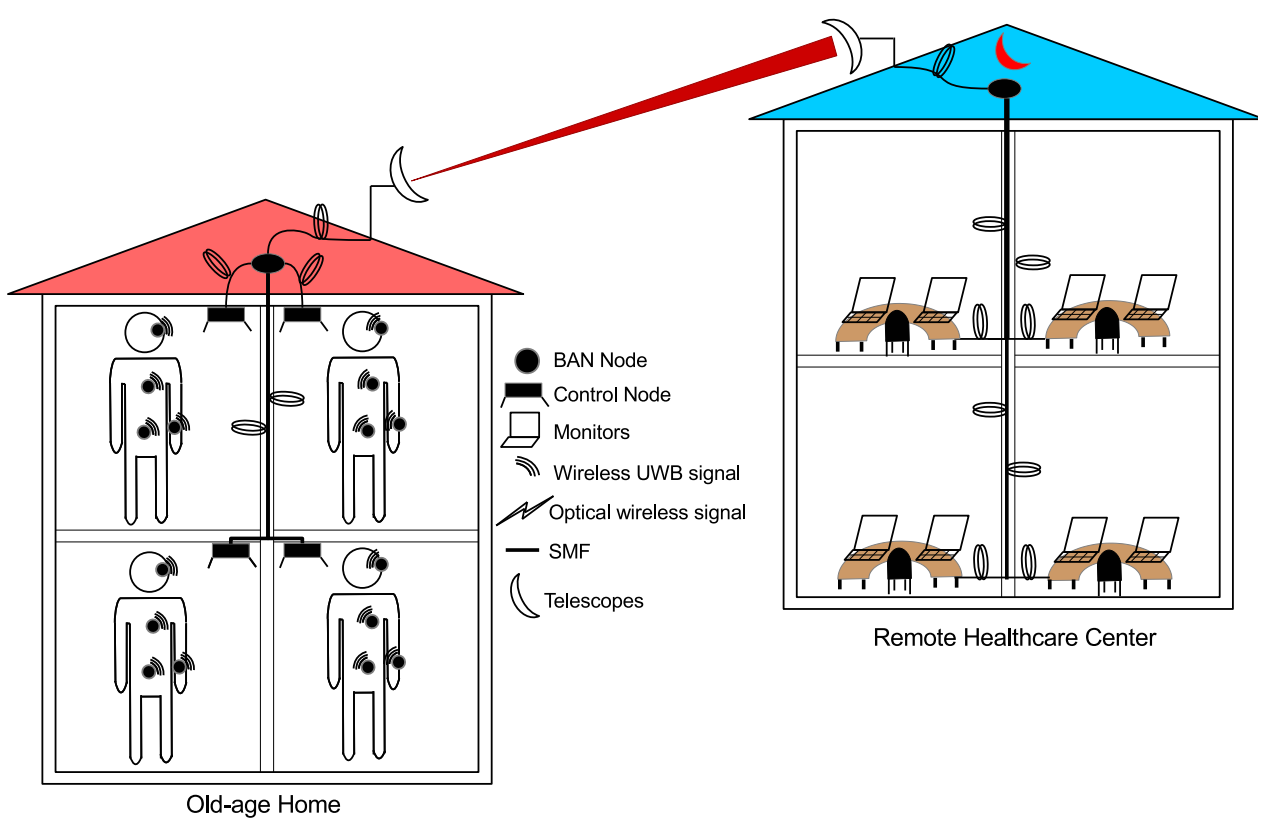

Fig. 1 Application scenario of proposed architecture, BAN: Body area network, UWB: Ultra-wideband, SMF: Single mode fiber

The application of our proposed OBAN architecture is best suited in oldage homes where elder citizens having multiple chronic diseases are living. The application scenario of the proposed OBAN architecture is shown in Fig. 1. It may be observed from the figure that elder citizens having multiple chronic diseases are living in their rooms located in an old-age home. We assume that each room has dimensions of $10 \mathrm{~m} \times 8 \mathrm{~m}$. Each patient is equipped with four on-body UWB-BAN nodes sensing pulse rate, body temperature, electrocardiogram (ECG) and electroencephalogram (EEG) activities of the patients. UWB-BAN nodes are transmitting real time data of aforementioned vital signs of the patients in the form of low power spectral density (PSD) UWB signals towards control nodes 
located at a fixed position which is $8 \mathrm{~m}$ high from room floor. The UWB-BAN nodes helps in minimizing the EMI as well as the RF exposure to human body. Each node is transmitting simultaneously at a different radio frequency to avoid the interference among UWB signals at control nodes. The optical signals of all control nodes are combined and then transmitted over FSO link towards remote health-care center. FSO link provides a cost efficient solution to implement the proposed architecture as compared to RF and optical fiber based BANs. As FSO links are prone to eavesdropping, privacy and multiple access in transmission is achieved using SAC-OCDMA scheme which is implemented at control nodes. The combined signal is decoded at remote health-care center and after optical to electrical conversion, the patient data is processed and interpreted by the nursing staff. The application scenario promises a comprehensive and alternative e-health and tele-medicine platform for optimum nursing and look after of elder citizens living in old-age homes. The application scenario not only provides optimum nursing to elder citizens in old-age homes but also minimizes the health-care expenditures including permanent stationing of nursing staff at old-age homes, regular visits of physicians or elder citizens visits to cardiologist or neurologist etc.

\section{Code Construction}

The proposed DW-ZCC code is developed by considering three performance parameters of DW-ZCC which includes code length (L), hamming weight $(\mathrm{W})$ and cross-correlation $\left(C_{\max }\right)$ between the adjacent codes represented by $\mathrm{X}$ and $\mathrm{Y}$. The cross-correlation can be defined mathematically as.

$$
C_{\max }=\sum_{i=1}^{L} x_{i} y_{i}=0
$$

In Eq. 1, $\mathrm{X}$ and $\mathrm{Y}$ are two adjacent code words where $X=x_{1}, x_{2} \ldots \ldots x_{L}$ and $Y=y_{1}, y_{2} \ldots . y_{L} . x_{i}$ and $y_{i}$ are bit values of respective code sequences. Now the basic matrix with $\mathrm{U}$ code sequences of length $\mathrm{L}$ can be written as:

$$
Z_{B}=\left[\begin{array}{llllllll}
1 & 1 & 0 & 0 & 0 & 0 & 0 & 0 \\
0 & 0 & 1 & 1 & 0 & 0 & 0 & 0 \\
0 & 0 & 0 & 0 & 1 & 1 & 0 & 0 \\
0 & 0 & 0 & 0 & 0 & 0 & 1 & 1
\end{array}\right]_{U \times L}
$$

Where $\mathrm{U}$ represents the number of nodes. From the basic matrix $Z_{B}$, large number of nodes can be accommodated by using a simple mapping technique such as:

$$
Z_{B}=\left[\begin{array}{cc}
Z_{B} & 0 \\
0 & Z_{B}
\end{array}\right]_{8 \times 16}
$$

Table 1 shows the proposed code with $U=4$ and $W=2$, where PoS represent the position of 1 in each code sequence.

It can be observed that there are no overlapping bits between the adjacent codes and throughout the $Z_{B}$ code matrix. Moreover, the association of the number of nodes and weight yields the total code length as $L_{t}=U \times L$. 
Table 1 PROPOSED CODE $(U=4, W=2)$

\begin{tabular}{llllllllll}
\hline \multirow{2}{*}{ Nodes } & \multicolumn{8}{c}{ Wavelengths } & \multirow{2}{*}{ Positions } \\
\cline { 2 - 7 } & $\lambda_{1}$ & $\lambda_{2}$ & $\lambda_{3}$ & $\lambda_{4}$ & $\lambda_{5}$ & $\lambda_{6}$ & $\lambda_{7}$ & $\lambda_{8}$ & \\
\hline Node\#1 & 1 & 1 & 0 & 0 & 0 & 0 & 0 & 0 & $\operatorname{PoS}_{1}=[1,2]$ \\
Node\#2 & 0 & 0 & 1 & 1 & 0 & 0 & 0 & 0 & $\operatorname{PoS}_{1}=[3,4]$ \\
Node\#3 & 0 & 0 & 0 & 0 & 1 & 1 & 0 & 0 & $\operatorname{PoS}_{1}=[5,6]$ \\
Node\#4 & 0 & 0 & 0 & 0 & 0 & 0 & 1 & 1 & $P o S_{1}=[7,8]$ \\
\hline
\end{tabular}

\subsection{Code Properties}

In order to determine an efficient structure of the encoder and decoder modules, and recover the intended spectrum with desired correlation properties, it is of primal importance to observe the correlation between code sequences of the proposed DW-ZCC code. It is evident from Table 1 that no over-lapping bits exist between the codes. Therefore, correlation properties for the proposed setup can be written as.

$$
C_{\text {max }}=\sum_{i=1}^{L} Z_{x}(i) Z_{y}(i)=\{W ; x=y, 0 ; x \neq y\}
$$

Here $Z_{x}(i)$ and $Z_{y}(i)$ denotes the $i^{\text {th }}$ element of the $\mathrm{X}$ and $\mathrm{Y}$ code sequences, respectively. It can be observed that zero cross-correlation exists between code sequences of the proposed codes. Therefore, direct detection can be adapted to recover the intended spectrum with maximum auto and minimum cross-correlation. In addition, position of $1 \mathrm{~s}$ in proposed code exists in pairs that significantly simplifies the implementation of encoder and decoder along with the allocation of desired spectrum.

\section{Proposed Architecture}

The architecture of the proposed OBAN is shown in Fig. 2. The working principle of the architecture has been explained in detail in coming sections.

\subsection{UWB-BAN Nodes}

As mentioned earlier, multiple patients with limited mobility are inhabiting in rooms having dimensions of $10 \mathrm{~m} \times 8 \mathrm{~m}$ situated in an old-age home, as shown in Fig. 1. For simplicity, we consider only one patient which is equipped with four onbody UWB-BAN sensors recording the vital physiological data such as pulse rate, body temperature, ECG and EEG activity and simultaneously communicating with control node. Each UWB-BAN node is transmitting the patient physiological data simultaneously over AWGN channel at $30 \mathrm{Mbps}$ in the form of FCC compliant UWB mono-cycle pulses which are generated by taking the first order derivative of electrical Gaussian pulses. Each UWB-BAN node is transmitting at a different radio frequency to avoid interference at the control node. Therefore, the electrical bandpass filters (EBPFs) of UWB-BAN nodes shown in Fig. 2 are centered at $f_{C 1}=4 \mathrm{GHz}, f_{C 2}=4.5 \mathrm{GHz}, f_{C 3}=5 \mathrm{GHz}$ and $f_{C 4}=5.5 \mathrm{GHz}$. The time 


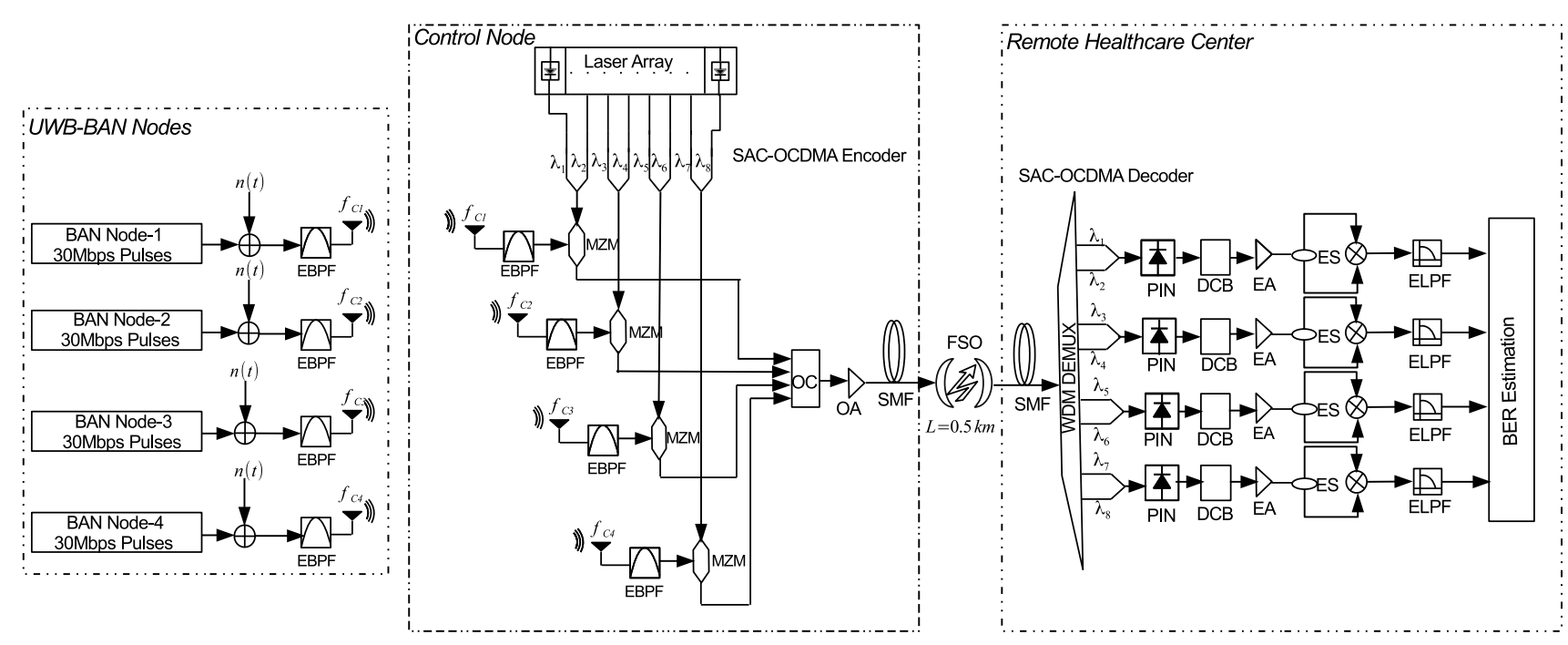

Fig. 2 Proposed architecture, BAN: Body area network, UWB: Ultra-wideband, SMF: Single mode fiber, FSO: Free space optics, EBPF: Electrical bandpass filter, OA: Optical amplifier, WDM-DEMUX: Wavelength division multiplexer, MZM: Mach zehnder modulator, OC: Optical coupler, PIN: Positive interinsic negative, DCB: DC block, EA: Electrical amplifier, ES: Electrical splitter, ELPF: Electrical lowpass filter

and frequency domain plots of UWB mono-cycle pulses generated by UWB-BAN nodes are shown in Fig. 3 and Fig. 4, respectively. In order to simulate the effect of AWGN channel on transmitted UWB signals, a white noise source represented by $n(t)$ following normal distribution is coupled with each UWB-BAN node, as shown in Fig. 2.

\subsection{Control Node}

Due to channel induced impairments, the received UWB mono-cycle pulses at control node are distorted. To encode the received UWB pulses of each node by using SAC-OCDMA scheme at control node, we use a CW laser array[14] generating eight wavelengths at same power level at its output port. These eight wavelengths are centered at $\lambda_{1}=1552.524 \mathrm{~nm}, \lambda_{2}=1551.720 \mathrm{~nm}, \lambda_{3}=1550.918 \mathrm{~nm}$, $\lambda_{4}=1550.116 \mathrm{~nm}, \lambda_{5}=1549.315 \mathrm{~nm}, \lambda_{6}=1548.514 \mathrm{~nm}, \lambda_{7}=1547.715 \mathrm{~nm}$ and $\lambda_{8}=1546.916 \mathrm{~nm}$. The combined spectrum of the eight wavelengths generated through a CW laser array that are being used for encoding and modulation, is shown in Fig. 5

\subsubsection{SAC-OCDMA Encoding}

Encoding operation for the DW-ZCC code is performed using the arrangement of optical couplers shown in Fig. 2. Since the CW laser array generates eight distinct 

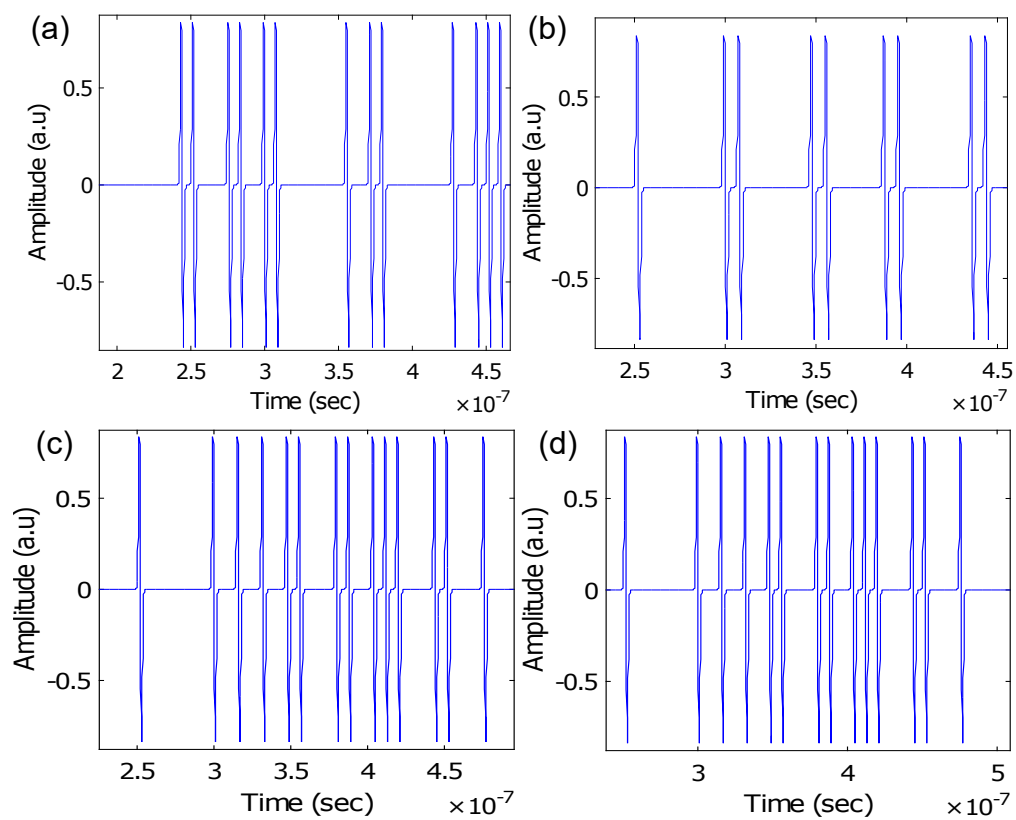

Fig. 3 Time domain plots of UWB mono-cycle pulses. (a) Node-1, (b) Node-2, (c) Node-3, (d) Node-4.
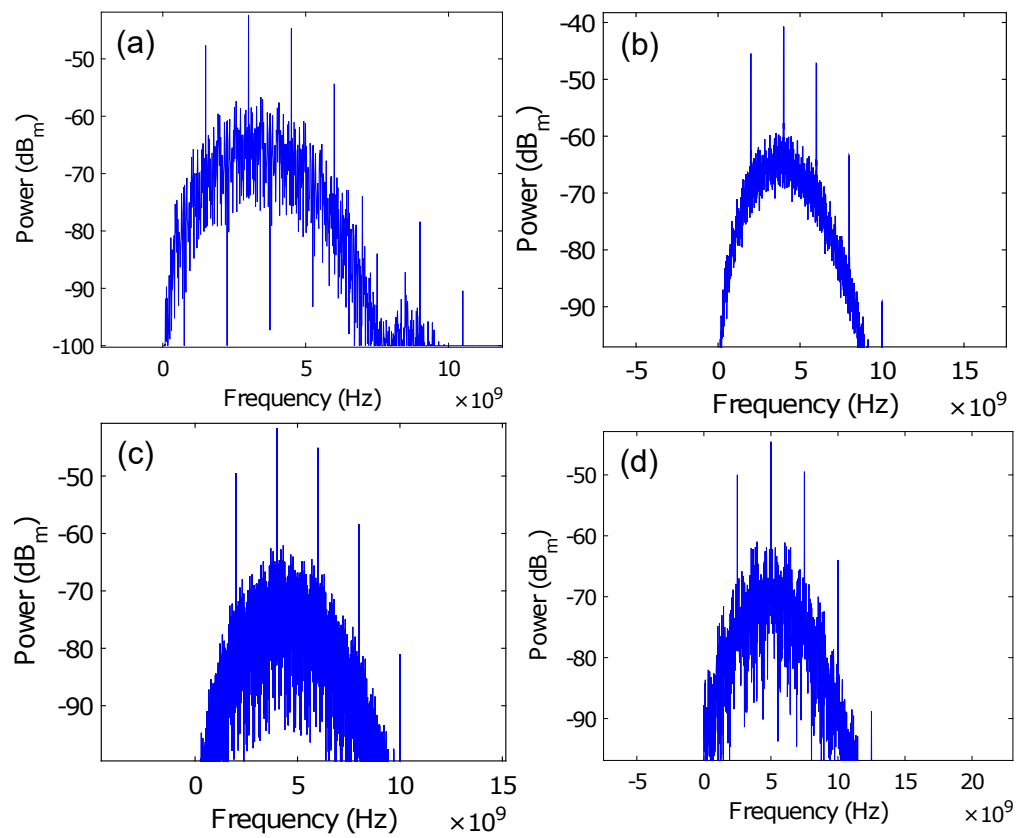

Fig. 4 Spectral plots of UWB mono-cycle pulses. (a) Node-1, (b) Node-2, (c) Node-3, (d) Node-4. 


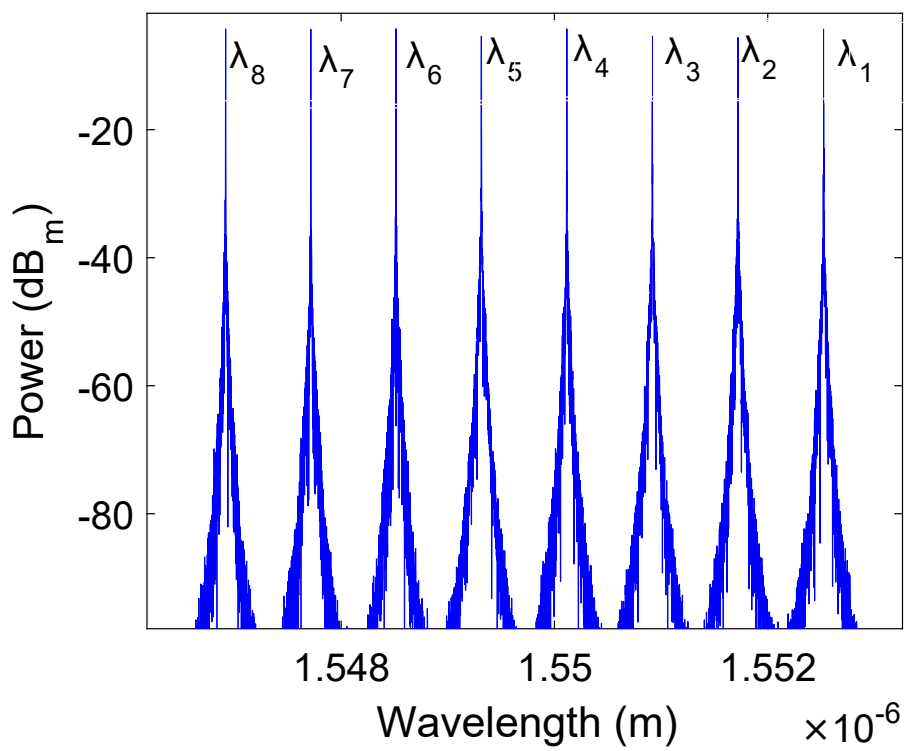

Fig. 5 Spectrum of the multiwavelength source.

wavelengths that are $0.8 \mathrm{~nm}$ apart, therefore, a simple combination of optical coupler is used as an encoder to combine the specific wavelength in reference to the proposed DW-ZCC code. For example for UWB-BAN node $\# 1, \lambda_{1}=1552.524 \mathrm{~nm}$ and $\lambda_{2}=1551.720 \mathrm{~nm}$ are combined to translate the binary 1's in DW-ZCC code into spectral representation as shown in Table 1. End-face of the encoder arrangement is connected to Mach-Zehnder Modulator (MZM) that is used to modulate the encoded signal through on-off keying (OOK) with the patient's physiological data transmitted by UWB-BAN node\#1 and received at the control node. After encoding operation, each 1 in the patient's physiological data is represented by a combination of two carriers centered as per the proposed code. Consequently, increasing the efficiency of the system with added benefits of built-in security to eavesdropping. In the same way, node\#2, node\#3 and node\#4 are encoded using SAC-OCDMA scheme and then OOK modulated with their respective patient physiological data signals as described earlier for UWB-BAN node\#1.

The full width at half maximum (FWHM) of the UWB mono-cycle pulses of each UWB-BAN nodes at the output of the MZMs after OOK modulation is around 125 ps. All four encoded optical signals after OOK modulation are combined using an optical coupler, as shown in Fig. 2. After suitable optical amplification, the combined optical signal is transmitted through a single mode fiber (SMF) having length of $3.5 \mathrm{~m}$ to a telescope that transmits it towards the remote health-care center over the FSO link of length $0.5 \mathrm{~km}$. The total power radiated by the transmitter telescope in free space is around $30 d B_{m}$. In this work, log-normal FSO channel model is used to model the atmospheric turbulence between control node and remote health-care center. The log-normal channel model is generally implemented for clear sky FSO links where turbulence is weak [15]. Therefore, the 
values of refractive index structure parameter $\left(C_{n}^{2}\right)$ and atmospheric attenuation $\left(\alpha_{a t m}\right)$ used in this simulation are chosen as $5 \times 10^{-16}$ and $3 \mathrm{~dB} / \mathrm{km}$, respectively.

\subsection{Remote Health-care Center}

At the remote health-care center, a receiver telescope receives the signal transmitted by the control node telescope, as shown in Fig. 2. The FSO transmitter and receiver telescopes employed in our simulation study have parameters similar to the commercial model G-2000 manufactured by CableFree [16]. The combined optical signal is impaired by turbulence and attenuation from the atmosphere. The mathematical model of the channel is discussed in Section 5.1 of the paper.

Table 2 Parameters of the transmitter and receiver Telescope (MODEL: G-2000, Manufacturer: CableFree

\begin{tabular}{lll}
\hline Sr. No & Parameters & values \\
\hline 1 & Diameter of Transmitter telescope & $5 \mathrm{~cm}$ \\
2 & Diameter of Receiver telescope & $20 \mathrm{~cm}$ \\
3 & Transmitter telescope apertures & 1 \\
4 & Receiver telescope apertures & 1 \\
5 & Material & GRIN lens \\
6 & Maximum transmission speed & $1.5 \mathrm{Gbps}$ \\
7 & Operating wavelength & $1550 \mathrm{~nm}$ \\
8 & Maximum working distance & $2 \mathrm{~km}$ \\
\hline
\end{tabular}

\subsubsection{SAC-OCDMA Decoding}

The encoded signal is given as input to SAC-OCDMA decoder through a SMF having length of $3.5 \mathrm{~m}$. The decoding operation has been performed by using the WDM-DEMUX arrangement shown in Fig. 2. Since the proposed DW-ZCC code bears zero cross-correlation between adjacent codes; therefore, the highly acknowledged direct detection is adapted to recover the intended spectrum. The filter arrangement in WDM-DEMUX is utilized to receive the desired spectrum as shown in Fig. 2. As a result, specific wavelengths corresponding to each code sequence are received and combined using an optical coupler. The proposed technique significantly simplifies the overall architecture with the added benefit of receiving the intended spectrum with maximum auto and minimum cross correlation. The output of each coupler is further given as input to a PIN photo-detector to convert the signal from optical to electrical domain. As zero cross-correlation exists between adjacent codes; therefore, no multiple access interference and accompanying optical beat interference is generated at the photo-detector during optical to electrical conversion that significantly elevates the quality of signal. The time domain plots of the photo-detected UWB mono-cycle pulses of each UWB-BAN node are shown in Fig. 6. 

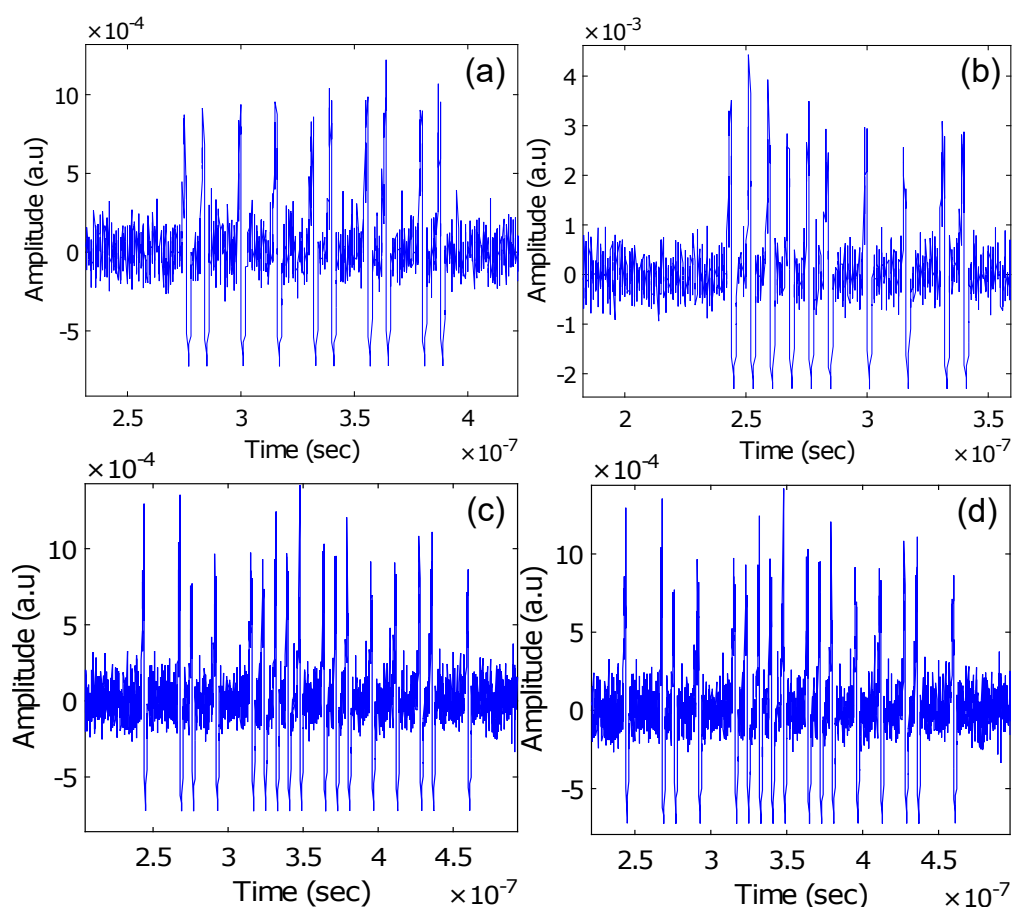

Fig. 6 Time domain plots of photo-detected UWB mono-cycle pulses. (a) Node-1, (b) Node-2, (c) Node-3, (d) Node-4.

The photo-detected UWB mono-cycle pulses of each UWB-BAN node obtained at the output of PIN photo-detectors are passed through a DC blocking circuit to remove DC off-set and then amplified using electrical amplifiers. The amplified signal is self-mixed, resulting in conversion of the patient physiological data from UWB mono-cycle pulses to Gaussian pulses by removal of the negative cycle due to multiplication [5]. The resulting electrical signal is low pass filtered using Gaussian filtering and forwarded to a BER estimator, as shown in Fig. 2. The summary of major simulation parameters for the setup of Fig. 2 is shown in Table 3.

\section{Mathematical Analysis of the Proposed Architecture}

To evaluate the BER of the signal received by the intended subscriber in this optical model of DW-ZCC based SAC-OCDMA system, the mathematical analysis of the proposed architecture utilizes log-normal channel model to determine the signal-to-noise ratios (SNRs) for back-to-back as well as for turbulence conditions. In the mathematical analysis, we are considering only those noise contributors that are being added in the system between SAC-OCDMA encoder and PIN photodetectors while the simulation results are being taken by considering all noise contributors between UWB-BAN nodes and PIN photo-detectors. 
Table 3 Major simulation parameters

\begin{tabular}{lll}
\hline Sr. No & Parameters & values \\
\hline 1 & Bit rate (per UWB-BAN node) & $30 \mathrm{Mbps}$ \\
2 & Transmitter telescope diameter & $5 \mathrm{~cm}$ \\
3 & Receiver telescope diameter & $20 \mathrm{~cm}$ \\
4 & Beam divergence & $2 \mathrm{mrad}$ \\
5 & Length of FSO link & $0.5 \mathrm{~km}$ \\
6 & Length of each SMF spool & $3.5 \mathrm{~m}$ \\
7 & Refractive index structure parameter & $5 \times 10^{-16}$ \\
8 & Atmospheric attenuation ( $\left.\alpha_{a t m}\right)$ of FSO link & $3 \mathrm{~dB} / \mathrm{km}^{-16}$ \\
9 & Responsitivity of PIN photo-detector & $0.9 \mathrm{~A} / \mathrm{W}$ \\
10 & Optical amplifier gain & $40 \mathrm{~dB}$ \\
11 & Optical amplifier noise figure & $4 \mathrm{~dB}$ \\
11 & Electrical amplifier gain & $25 \mathrm{~dB}$ \\
\hline
\end{tabular}

\subsection{Log-Normal Channel Model}

A FSO link induces mainly two types of impairments known as attenuation and atmospheric turbulence. Atmospheric turbulence is the consequence of variations in the atmospheric temperature and pressure along the path of the signal. It is a major cause of signal degradation and results in random variations in signal irradiance, commonly known as intensity scintillation. Various statistical channel models have been proposed to consider the effect of attenuation and atmospheric turbulence on the optical signal [17]. Some of the most commonly used channel models to induce atmospheric turbulence on the optical signals are the negative exponential, K-distribution, log-normal distribution, log-normal-Rician and Gamma-Gamma channel model [17]. To characterize weak turbulence conditions of a clear sky link, the log-normal distribution is employed [15]. The probability density function of received light intensity $I$ following a planar wave propagation in terms of variance of log-amplitude fluctuations $\left(\sigma_{x}^{2}\right)$ is given by [18]:

$$
p_{I}(I)=\frac{1}{2 I \sqrt{2 \pi \sigma_{x}^{2}}} \exp \left[-\frac{\ln \left(I / I_{o}\right)^{2}}{8 \sigma_{x}^{2}}\right],
$$

where $\sigma_{x}^{2}=0.307 C_{n}^{2} k^{7 / 6} L^{11 / 6}, L$ is the FSO link length in kilometers, $k=$ $2 \pi / \lambda$ is the wave number and $C_{n}^{2}$ represents the refractive index structure parameter whose values vary from $10^{-17}$ to $10^{-12}$ for weak turbulence to strong turbulence, respectively [18]. Even for a specific link, the refractive index structure parameter can vary over time due to the complex dynamics of the weather.

\subsection{BER Calculations}

The SNR for analysis of the proposed setup can be written as ratio of the average desired photo-current $I_{b}$ received by the intended subscriber to the power of 
different noise sources $i_{b n}^{2}$ generated throughout the system [10]:

$$
S N R=\left[\frac{I_{b}^{2}}{i_{b n}^{2}}\right]
$$

The average desired photo-current in Eq. 4 can also be written as [10]:

$$
I_{b}=\Re b W_{m} P_{s r},
$$

where $b \in\{0,1\}$ is value of the bit that represents the transmission of binary 1 or 0 by the intended user. $P_{s r}$ shows the power per chip at the receiving end and is equivalent to $\frac{P_{t x} D^{2} e^{-\alpha Y}}{N(\theta d)^{2}}$, where $P_{t x}$ is the transmitted power, $D$ is the aperture diameter of the receiving side telescope, $\alpha$ is the atmospheric attenuation, $d$ distance between transmitter and receiver side telescopes and $\theta$ is the beam divergence. In Eq. $5, \Re$ is the responsivity of the photo-detector that is used to convert the received signal from optical to electrical domain. $W_{m}$ represents power units in the number of chips for each user absorbed by the photo-detector. Since our proposed setup uses direct detection that recovers the non-overlapping spectral chips only; therefore, $W_{m}=W$ which indicates that maximum power units in recovered spectrum is absorbed by the photo-detector. The variance of the total noise power for the proposed DW-ZCC based SAC-OCDMA system can be written as the sum of noise sources generated throughout the system that primarily includes optical beat interference $i_{o b n}$, relative intensity noise $i_{r n}$, shot noise $i_{s n}$ and thermal noise $i_{t n}[10]$ :

$$
i_{b n}^{2}=i_{o b n}^{2}+i_{r n}^{2}+i_{s n}^{2}+i_{t n}^{2}
$$

As spectral direct detection technique recovers the intended spectrum, therefore, only the desired pulses will hit the photo-detector. Moreover, cross-correlation between the adjacent codes of the proposed DW-ZCC codes is equal to 0, therefore, the value of $i_{\text {obn }}^{2}=0$ for the proposed setup. Relative intensity noise $(R I N)$ is generated at the transmitter module of the desired signal. Moreover, all users in the proposed setup cause cross-talk with the desired user signal; therefore, power of the $R I N$ with $W m=W$ can be written as [19]:

$$
i_{r n}^{2}=R N\left(b W P_{s r}+x P_{c}\right)^{2} B_{e}
$$

Here, $R N$ is the noise factor with typical value between -130 and $-160 \mathrm{dBHz}^{-1}$, $B_{e}$ represents the electrical bandwidth, $P_{c}$ denotes the optical power in the crosstalk pulses, and $x$ is event of the whole interfering pulses from the possible users out of $U-1$ that transmits bit " 1 ". The average value of $x$ when the number of interfering users that are sending bit " 1 " at every chip is equal and can be written as $[10]$.

$$
x=\frac{W_{m}^{2}(U-1)}{2 L}
$$

Haphazard nature of the photons that are incident upon the PIN photo-diode generates random electrons that results in fluctuation of the photo-current. This phenomenon generates shot noise that is proportional to the incident current times $2 E B_{e}$. Mathematically, the shot noise can be written as $[10,13]$ : 


$$
i_{s n}^{2}=2 \Re E\left(b W_{m} P_{s r}+x P_{c}\right) B_{e}
$$

Here $E$ is the electron charge. Since direct detection at the intended subscriber recovers non-overlapping spectrum of the DW-ZCC code; therefore, $P_{c}=0$ as no cross-talk is observed between the intended and interfering user at the receiving photo-diode $[10,13]$. Consequently, the value of $i_{s n}^{2}$ becomes:

$$
i_{s n}^{2}=2 \Re E b W_{m} P_{s r} B_{e}
$$

The thermal noise generated at the receiving photo-diode of the intended user can be written as [10]:

$$
i_{t n}^{2}=\frac{4 K_{B} T B_{e}}{R_{L}}
$$

where $T, K_{B}$, and $R_{L}$ represents the temperature, Boltzmann constant, and load resistance, respectively. The total variance of the noise power becomes [10]:

$$
i_{b n}^{2}=i_{r n}^{2}+i_{s n}^{2}+i_{t n}^{2}
$$

If the decision of the received bit is carried out by comparing the total current of the received signal with a threshold current $I_{T}$, then BER of the received optical signal can be calculated as [10]:

$$
\operatorname{BER}_{O}(I)=Q\left(\frac{I_{1}-I_{0}}{i_{n 1}+i_{n 0}}\right),
$$

where $I_{1}$, and $i_{n 1}$ is the total signal current and noise power for bit " 1 ", and $I_{0}$, and $i_{n 0}$ is the total signal current and noise power for bit " 0 ", respectively. The total BER of the encoded signal that is transmitted over the log-normal turbulent channel can be written as $[10,13,18]$ :

$$
B E R=\int_{0}^{\infty} B E R_{0}(I) p_{I}(I) d I
$$

Here $p_{I}(I)$ is the probability density function of the log-normal channel that is used under weak turbulence conditions to model the intensity fluctuations of the received signal at the photo-detector. The BER in Eq. 14 is used to calculate performance of the proposed system, in terms of quality of the received signal at the intended photo-diode.

\section{Performance Analysis}

The UWB signals received at the remote health-care unit after being transmitted from the control node and propagation through the log-normal channel are analyzed for BER performance. The eye-diagrams of the Gaussian-shaped electrical signal at the output of the electrical bandpass filters shown in Fig. 2, are used to statistically calculate the BER The optical power of the signals received at the PIN photo-detector is varied with the help of an optical attenuator to observe the effect on the BER of the UWB mono-cycle pulses. Fig. 7 shows the BER versus received optical power plots obtained while considering weak turbulence regime 


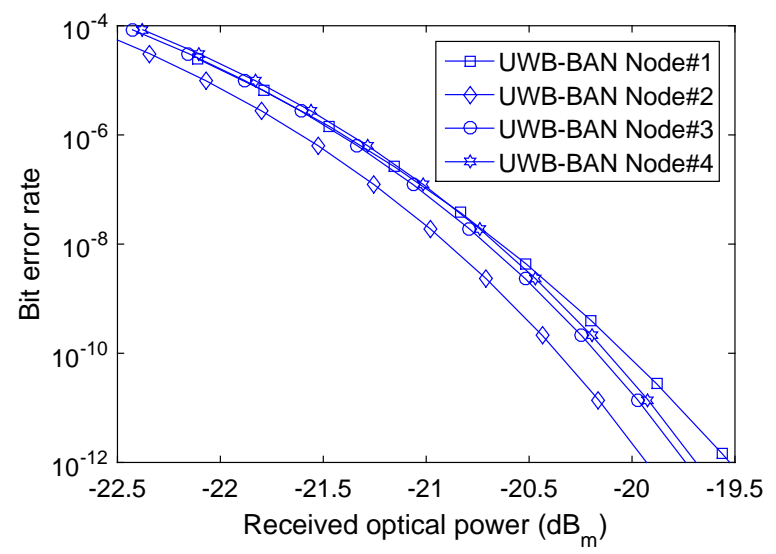

Fig. 7 BER versus received optical power plots for UWB-BAN nodes

which is specific to log-normal channel model. Receiver sensitivity is defined as the minimum optical power required to achieve a BER of $10^{-9}[20]$. The minimum value of receiver sensitivity is around $-20.5 d B_{m}$ which is obtained for UWB-BAN node-2. Its maximum value is $-20.25 d B_{m}$ which is obtained for UWB-BAN node1. Small variations in receiver sensitivity are observed among the four UWB-BAN nodes. Overall, the four UWB-BAN nodes give acceptable BER values indicating the suitability of our proposed technique for employment at health-care centers.

\section{Conclusion}

A low transmit power, low cost and secure OBAN composed of four on-body UWBBAN nodes, each transmitting the vital physiological data of patients at data rate of $30 \mathrm{Mbps}$ to remote health-care unit over FSO link is proposed. Security and multiple access is achieved through SAC-OCDMA scheme implemented by multiple wavelengths generated using a CW laser array. The performance of the proposed UWB-BAN architecture is analyzed with the help BER calculations. The proposed architecture gives good BER results with added advantages of low complexity, low cost and security.

\section{Conflicts of interest/Competing interests}

The authors have no conflict of interest/Competing interest for this submission.

\section{Code availability}

The authors are willing to provide on request the complete simulation file for this design. 


\section{References}

1. A. Astrin, "IEEE standard for local and metropolitan area networks part 15.6: Wireless body area networks," IEEE Std 802.15. 6, 2012.

2. L. Chevalier, S. Sahuguede, and A. Julien-Vergonjanne, "Performance evaluation of wireless optical communication for mobile body area network scenario with blocking effects," IET Optoelectronics, vol. 9, no. 5, pp. 211-217, 2015.

3. F. Hu, Y. Xiao, and Q. Hao, "Congestion-aware, loss-resilient bio-monitoring sensor networking for mobile health applications," IEEE Journal on Selected Areas in Communications, vol. 27 , no. 4, pp. 450-465, 2009.

4. M. Periyasam and R. Dhanasekaran, "Electromagnetic interference on critical medical equipments by RF devices," in 2013 International Conference on Communication and Signal Processing. IEEE, 2013, pp. 78-82.

5. J. Mirza, S. Ghafoor, and A. Hussain, "All-optical regenerative technique for width-tunable ultra-wideband signal generation," Photonic Network Communications, vol. 38, no. 1, pp. 98-107, Aug 2019.

6. D. Killinger, "Free space optics for laser communication through the air," Optics and Photonics News, vol. 13, no. 10, pp. 36-42, 2002.

7. H. Willebrand and B. S. Ghuman, Free space optics: enabling optical connectivity in today's networks. SAMS publishing, 2002.

8. L. Chevalier, S. Sahuguede, and A. Julien-Vergonjanne, "Optical wireless links as an alternative to radio-frequency for medical body area networks," IEEE Journal on Selected Areas in Communications, vol. 33, no. 9, pp. 2002-2010, 2015.

9. L. Chevalier, S. Sahuguede, A. Julien-Vergonjanne, P. Combeau, and L. Aveneau, "Investigation of wireless optical technology for communication between on-body nodes," in 2013 2nd International Workshop on Optical Wireless Communications (IWOW). IEEE, 2013, pp. $79-83$.

10. M. Moghaddasi, S. Seyedzadeh, I. Glesk, G. Lakshminarayana, and S. B. A. Anas, "DWZCC code based on SAC-OCDMA deploying multi-wavelength laser source for wireless optical networks," Optical and Quantum Electronics, vol. 49, no. 12, p. 393, 2017.

11. W. A. Imtiaz, "Design of high-capacity spectral amplitude coding OCDMA system with single photo-diode detection technique," Arabian Journal for Science and Engineering, vol. 43, no. 6, pp. 2769-2777, 2018.

12. A. Jurado-Navas, T. R. Raddo, J. M. Garrido-Balsells, B.-H. V. Borges, J. J. V. Olmos, and I. T. Monroy, "Hybrid optical CDMA-FSO communications network under spatially correlated gamma-gamma scintillation," Optics express, vol. 24, no. 15, pp. 16799-16814, 2016.

13. M. Moghaddasi, G. Mamdoohi, A. S. M. Noor, M. A. Mahdi, and S. B. A. Anas, "Development of SAC-OCDMA in FSO with multi-wavelength laser source," Optics Communications, vol. 356, pp. 282-289, 2015.

14. J. Yao, "Photonics to the rescue: A fresh look at microwave photonic filters," IEEE Microwave Magazine, vol. 16, no. 8, pp. 46-60, 2015.

15. E. J. Lee and V. W. Chan, "Part 1: Optical communication over the clear turbulent atmospheric channel using diversity," IEEE journal on selected areas in communications, vol. 22, no. 9, pp. 1896-1906, 2004.

16. J. Mirza, S. Ghafoor, and A. Hussain, "All-optical generation and transmission of multiple ultrawideband signals over free space optical link," Optical Engineering, vol. 58, no. 5, p. $056103,2019$.

17. L. C. Andrews and R. L. Phillips, Laser beam propagation through random media. SPIE press Bellingham, WA, 2005, vol. 152.

18. K. Davaslioglu, E. Cagiral, and M. Koca, "Free space optical ultra-wideband communications over atmospheric turbulence channels," Optics express, vol. 18, no. 16, pp. 16618-16627, 2010 .

19. S. M, Optical Fiber Communications: Principles and Practice. Pearson Education, 2009. [Online]. Available: https://books.google.com.pk/books?id=ok0XX-3MgMoC

20. J. Mirza, S. Ghafoor, and A. Hussain, "All-optical 2R-regeneration and continuous wave to pulsed signal wavelength conversion based on fiber nonlinearity," Optical and Quantum Electronics, vol. 50, no. 10, p. 366, 2018. 


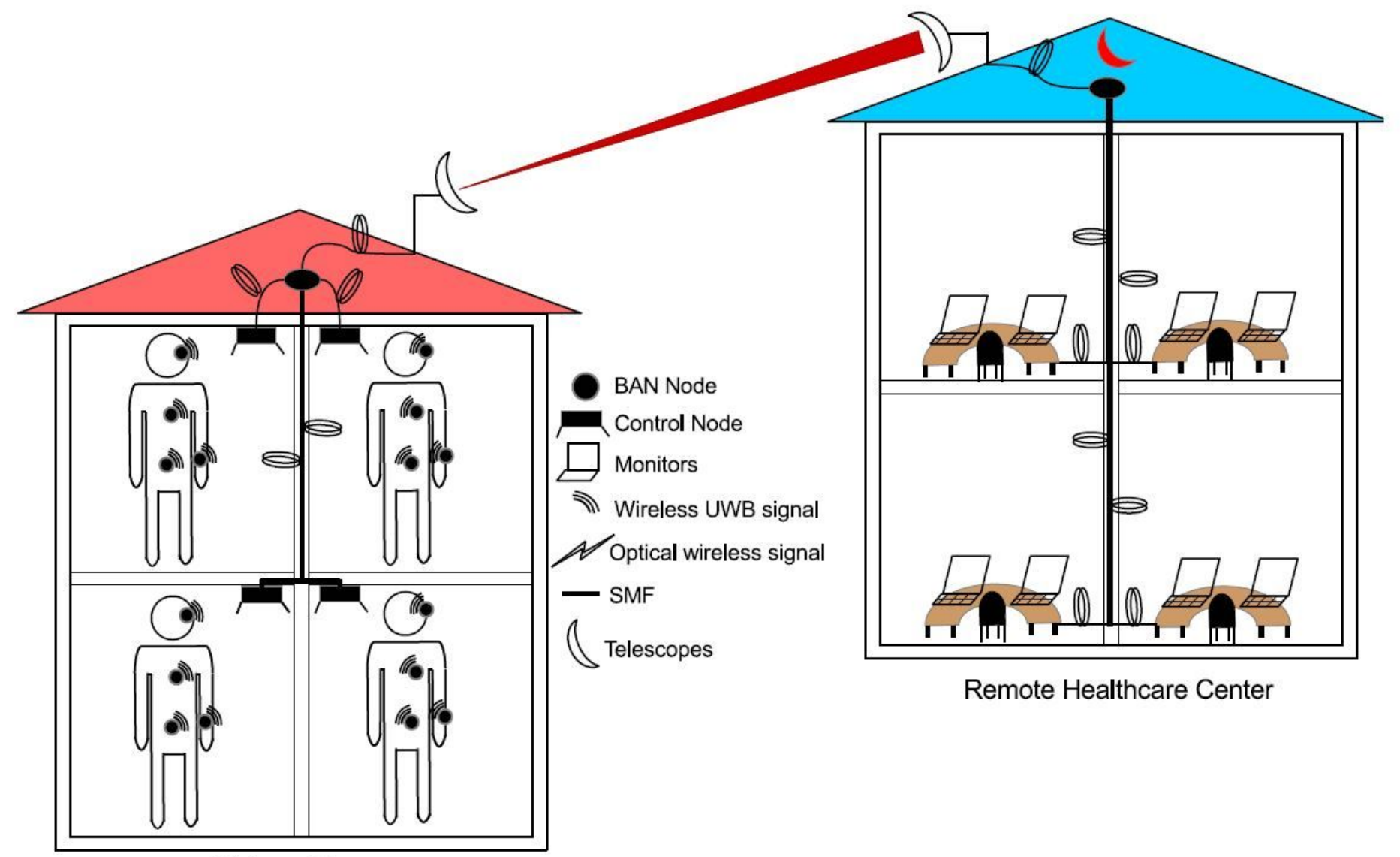

Old-age Home

\section{Figure 1}

Application scenario of proposed architecture, BAN: Body area network, UWB: Ultra-wideband, SMF: Single mode ber

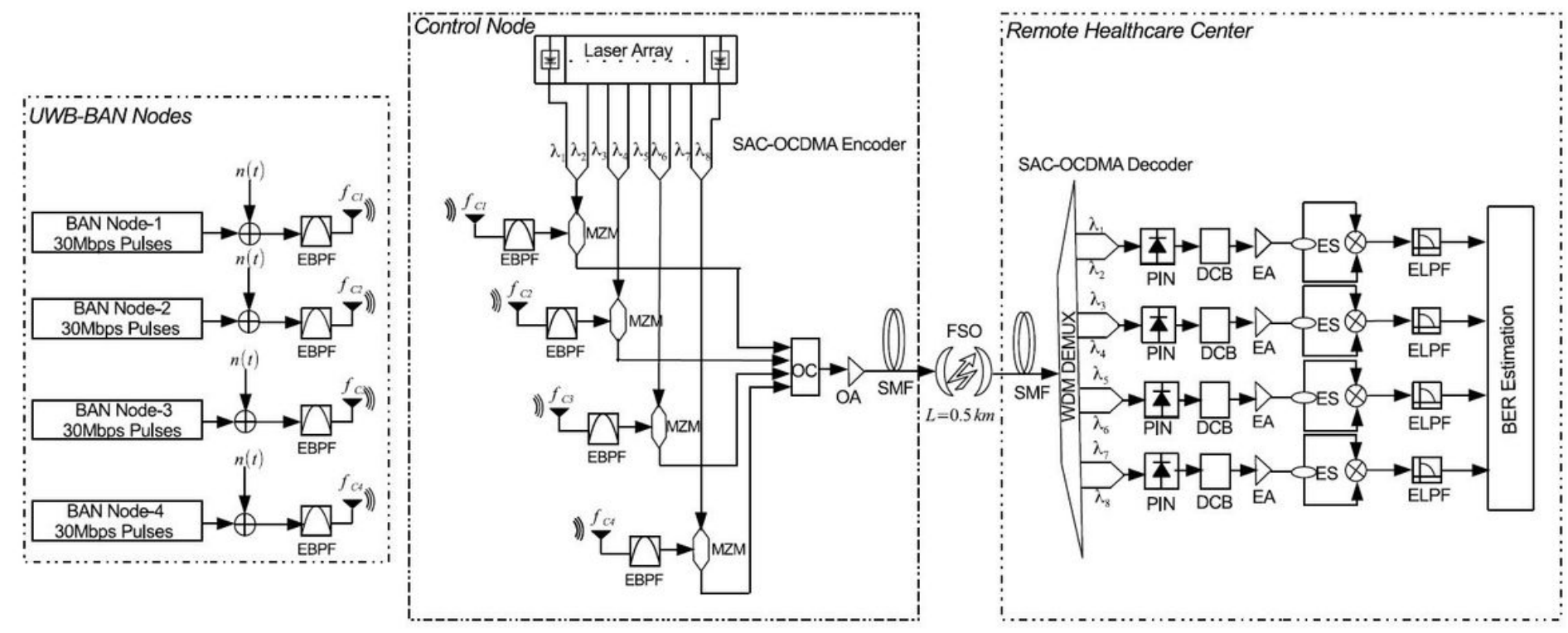


Figure 2

Proposed architecture, BAN: Body area network, UWB: Ultra-wideband, SMF: Single mode ber, FSO: Free space optics, EBPF: Electrical bandpass Iter, OA: Optical amplier, WDM-DEMUX: Wavelength division multiplexer, MZM: Mach zehnder modu- lator, OC: Optical coupler, PIN: Positive interinsic negative, DCB: DC block, EA: Electrical amplier, ES: Electrical splitter, ELPF: Electrical lowpass Iter
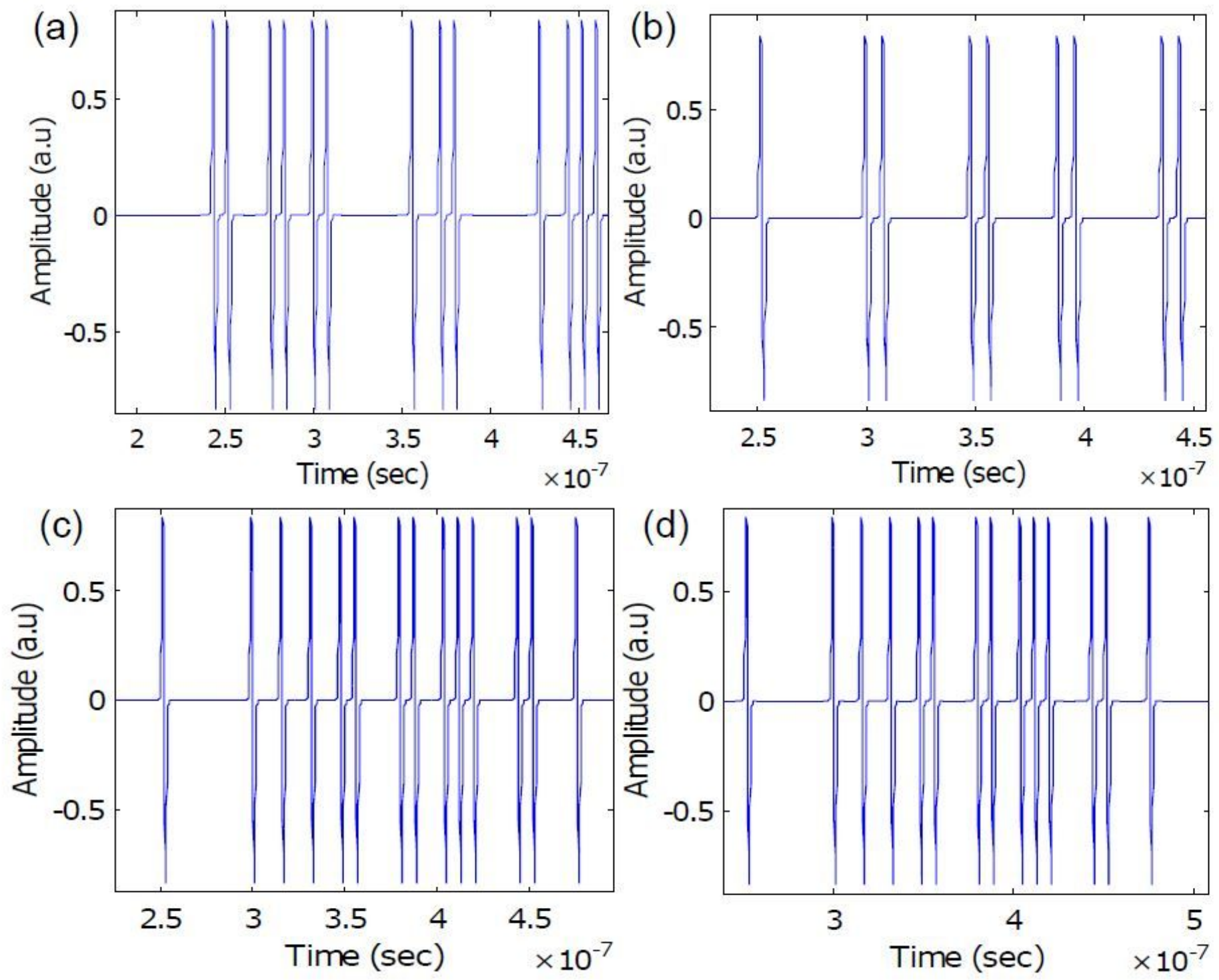

Figure 3

Time domain plots of UWB mono-cycle pulses. (a) Node-1, (b) Node-2, (c) Node-3, (d) Node-4. 

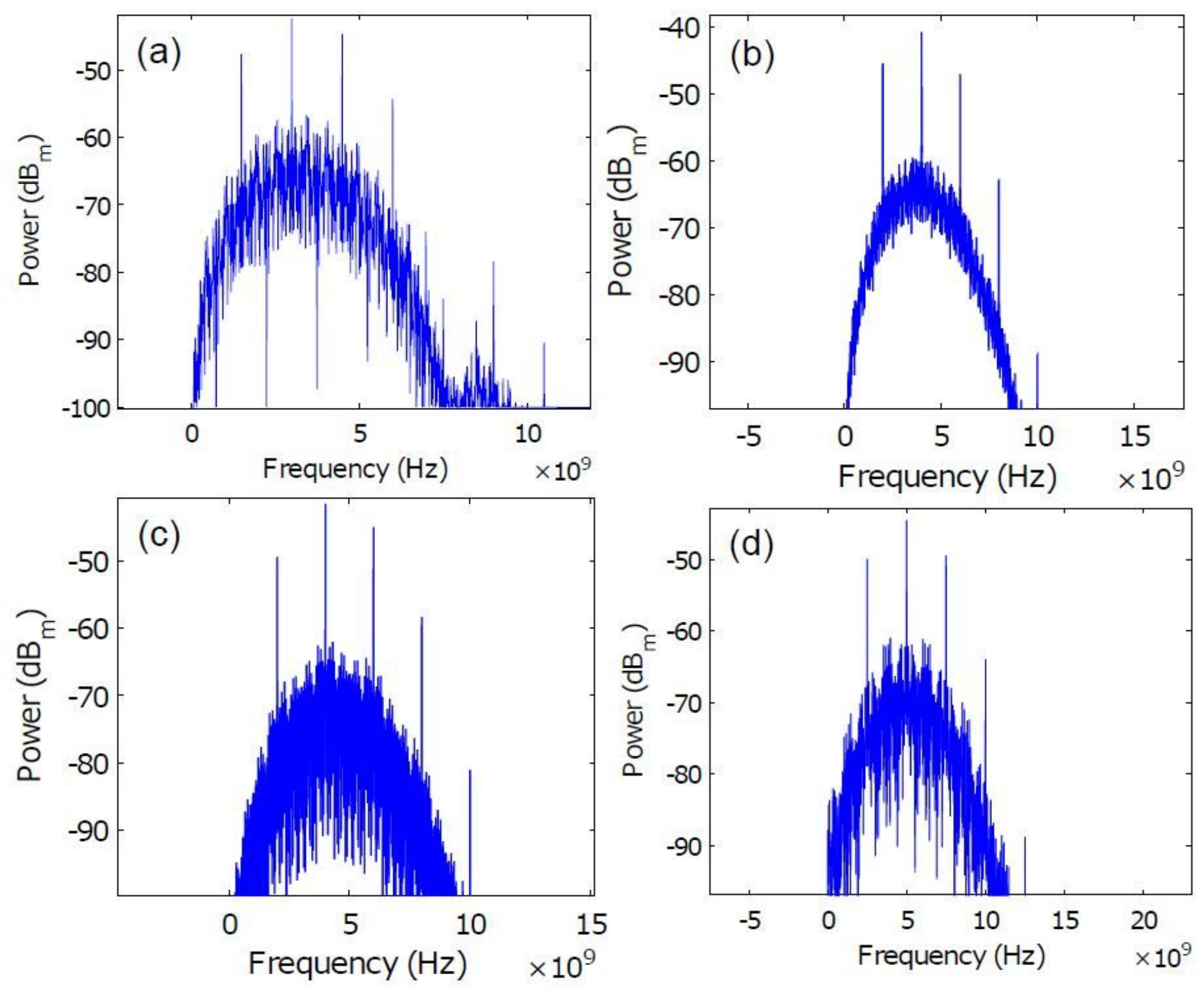

Figure 4

Spectral plots of UWB mono-cycle pulses. (a) Node-1, (b) Node-2, (c) Node-3, (d) Node-4. 


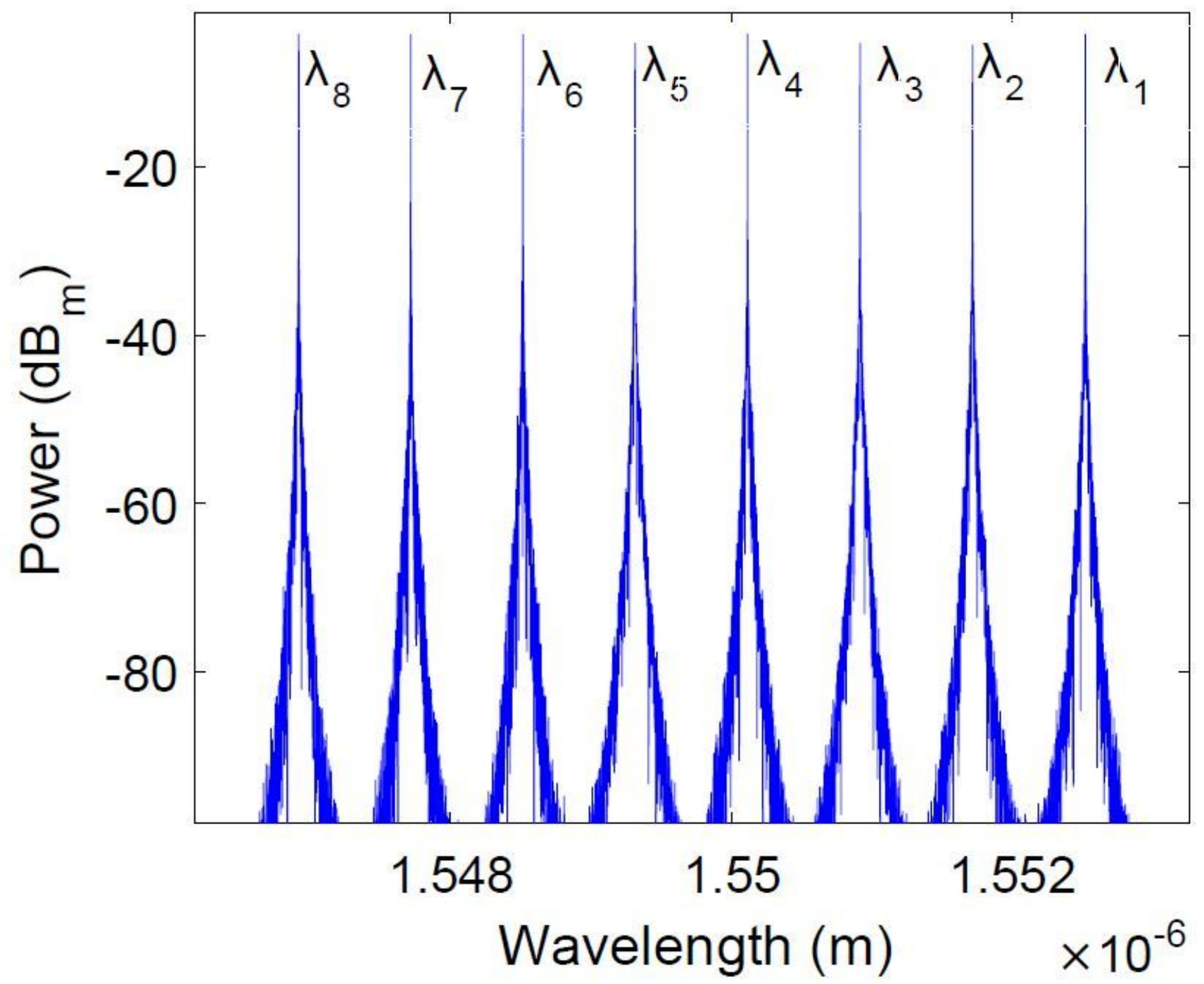

Figure 5

Spectrum of the multiwavelength source. 

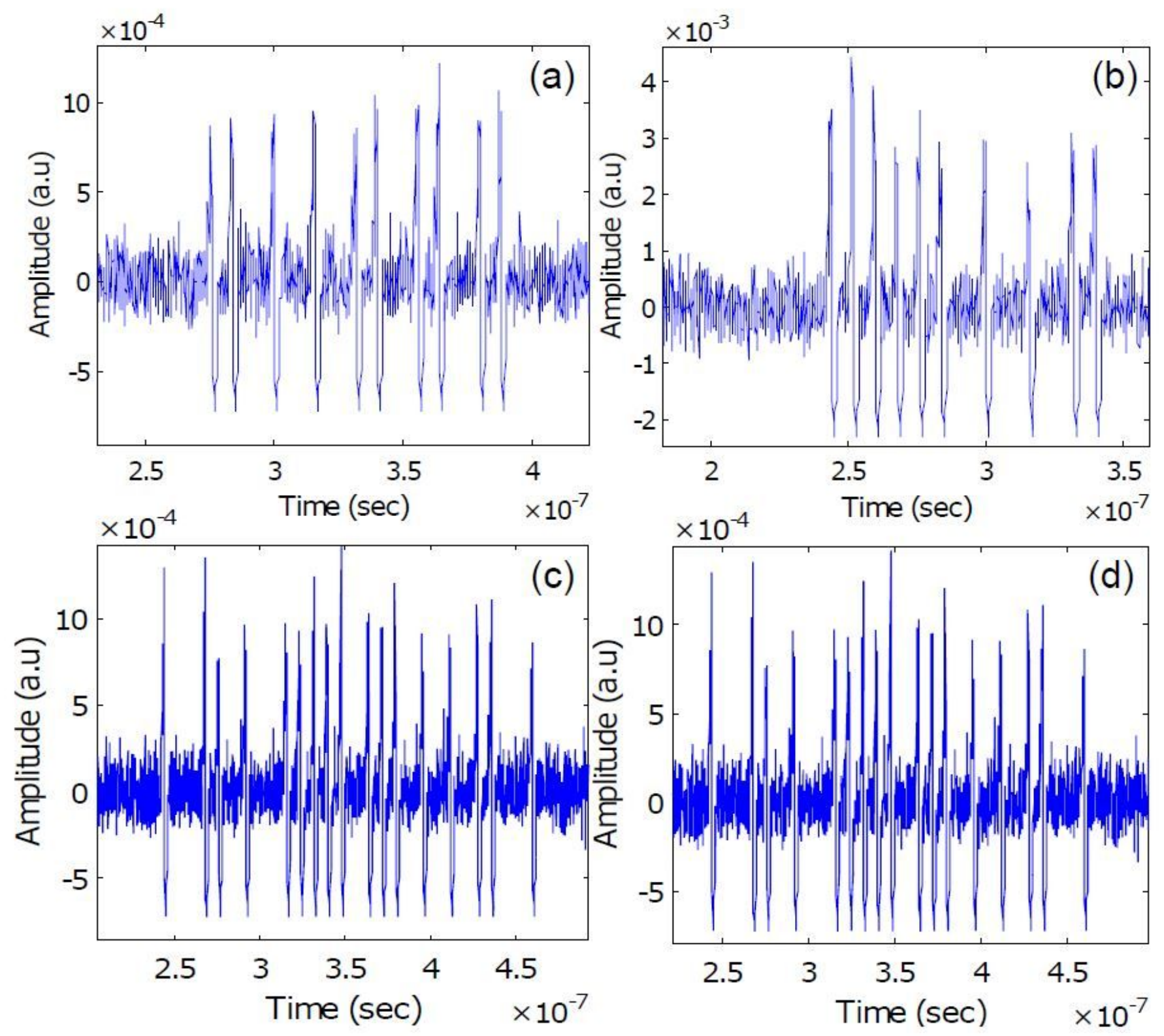

Figure 6

Time domain plots of photo-detected UWB mono-cycle pulses. (a) Node-1, (b) Node-2, (c) Node-3, (d) Node-4. 


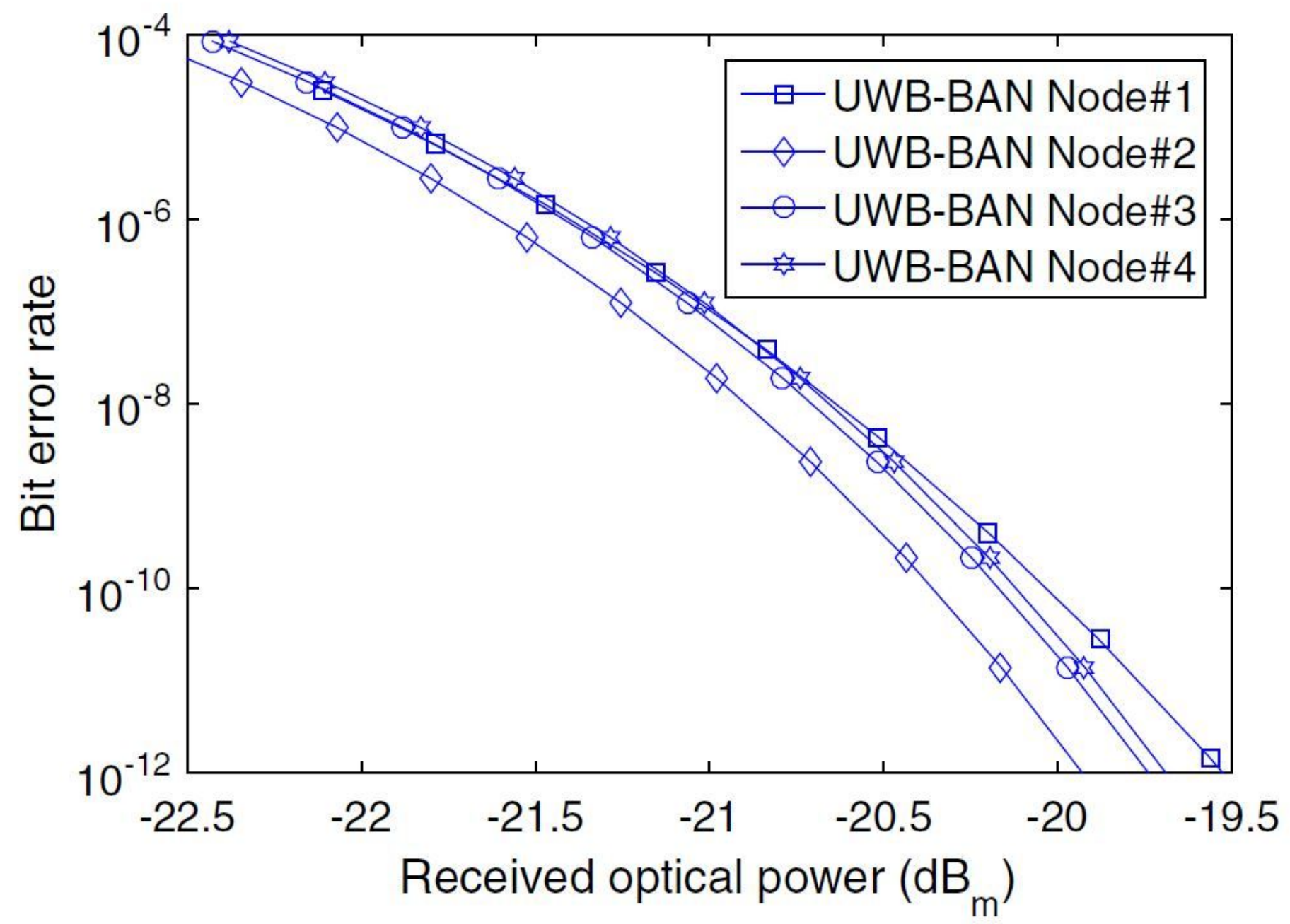

Figure 7

BER versus received optical power plots for UWB-BAN nodes 